\section{CASES OF OVARIOTOMY, WITH SOME REMARKS UPON THE OPERATION.}

By Wm. Warren Greene, M.D., Professor of Surgery in the Medical School of Maine and in the University of Michigan.

There is no occasion at the present day for multiplying reports of ovariotomy, unless the cases possess some unusual interest. Therefore, in presenting the following cases, which, eight in number, constitute all the operations that have performed up to this time, I shall avoid details, except they be of special value.

CASE I.-Miss —- aged 16. I first saw her with her physician, Dr. H. S. Lucas, of Chester, Mass., in December, 1862. She had been a healthy girl until two years before, at which time menstruation was established, and soon after she noticed an abdominal enlargement. She could not tell whether it began more upon one side than the other, but thought "both alike." This gradually increased, without pain or tenderness, or any marked impairment of the general heaith, until the spring of 1862 . At this time it increased rapidly, and even before the distention was extreme, her health failed quite suddenly. She lost strength and appetite, and suffered great derangement of the digestive organs. A marked feature was, also, an unusually rapid pulse, which Dr. Lucas assured me was 140 per minute, whereas its normal rate was below 80. Dr. L. tapped her at this time, drawing off " about three pailfuls of syrupy, molasses-colored fluid." The operation was followed by immediate relief of all unpleasant symptoms, appetite and strength returned, the pulse fell to its natural standard, and in a few.weeks she was in excellent health.

By the following September, the abdomen had again enlarged sufficiently to reproduce the symptoms above described, which were as speedily relieved by paracentesis as in the first instance, a tluid of similar character, though in less quantity, having been withdrawn.

I found her, in the following December, with all the evidences of a multilocular ovarian cyst, and with the same derangement of the general health as had preceded the two previous tappings. The heart's action was very rapid and feeble, and yet the enlargement was not nearly as great as is frequently seen when the action is little if at all affected by the pressure. My notes of the case do not include a statement of the quantity of liquid at this time evacuated, but I think the amount was about eighteen pounds. This was again followed by speedy restoration to health.

Having explained to herself and friends the nature of her case, and her chances with and without an operation, she was left, with the advice that in case she elected it, excision should be performed before the re-accumulation was sufficient to produce much general disturbance. In eight weeks, the sac had re-filled sufficiently to disturb the stomach and heart, and she decided upon an operation. This I made in the presence and with the assistance of Drs. H. S. Lucas, A. M. Smith, and F. K. Paddock.

Ether being administered, she was placed, supine, upon a table in a room the temperature of which was $80^{\circ} \mathrm{Fahr}$, and the air was kept constantly moist by steam. Standing upon the patient's right, I made an incision, with a common scalpel, in the me dian line, from above the umbilicus to the pubes. This was afterwards extended nearly to the ensiform cartilage. The tumor was readily exposed, and the principal cyst, seized and steadied by a tenaculum, was emptied of its fluid contents, which resembled that removed in the former tappings. There remained a fleshy mass filled with small cysts containing thick albuminous jelly, the whole being so large as to require the extension of the first incision above referred to. Adhesions of moderate strength existed over a considerable portion of the abdominal parietes, and also to the lower border of the stomach and liver, but none were sufficiently firm to prevent a ready separation of the tumor. The pedicle was found to be the right broad ligament. This was transfixed with a needle armed with a double ligature, and each half tied in the following manner, which I describe with some particularity, for a reason which will be apparent further on. I had turned the mass out of the abdomen towards myself, and while partially supporting it, tied one half of the pedicle. My friend, Dr. - who stood opposite, said, "Let me tie the other half, Doctor, I can reach it more easily than you" ; and, in violation of a rule from which $I$ have not since departed, I reluctantly allowed him to do so. As he tightened the knot, I noticed, what always makes me apprehensive, that he did so with a wriggling motion of the hands. This motion is often seen, and is made, I suppose, with the idea that thus the knot is more effectually tightened, whereas the effect, so far as any is produced, is almost invariably to loosen it. I said, "Doctor, are you sure that is tight?" He assured me 
that it was, and I divided the pedicle and removed the tumor, which woighed, contents included, forty.pounds. The ligatures were then carried through an opening made in the posterior cul de sac of the vagina down through that canal, and fastened to the thigh with adhesive plaster. During the entire operation, steady and even compression of the abdominal walls had been maintained by assistants, and my hands:and spanges kept constantly moist with artificial serum at blood-heat. After the cavity was cleansed, a considerable quantity of this fluid was poured in and allowed to drain off through the vagina. The external wound was then closed by interrupted sutures of silver wire, which included the entire thickness of the parietes, except the peritoneum. These were placed half an inch apart. A light compress being placed along the line of the wound, a swathe was applied and she placed in bed wrapped in warm blankets, with bottles of hot water around the extremities.

The patient recovered readily from the anæsthesia, and was in excellent condition. She took.a moderate dose of morphia, which was repeated pro re nata, but a very small amount being required to control pain.

I saw her the next day (Friday) at noon, and found her still in good condition, no symptoms of peritonitis presenting. She had slept quietly the greater part of the night, and was very cheerful and hopeful. On Saturday evening, I received a telegram from Dr. Lucas, saying, " - is doing splendidly ; she will get well." On Sabbath evening, I received another despatch, asking me to visit the lady as soon as possible. $A$ drive of twenty miles over terrible roads .was not very rapidly accomplished, and when I arrived, at 3 o'clock, Monday morning, the patient had been dead two hours. I learned that she remained entirely comfortable till late Sunday afternoon, when she began to be restless and anxious, complaining of slight abdominal uneasiness; from this time she grew gradually weak, pale, complained of faintness, sank and died. Dr. Lucas and myself were both of the opinion that she had died of hæmorrhage, and Dr. L. remarked that he had not "felt easy about the ligature that Dr. - tied." We examined the body, Dr. Paddock, then medical student, assisting. The external wound had united by first intention throughout. The abdomen was filled with blood, which, upon examination, was found to have issued from the half of the pedicle that we had suspected, the ligature having loosened, so as to admit the handle of the scalpel between it and the pedicle. I have the specimen. Not a sign of peritonitis appeared. The ligature of the other half was separating kindly, the stump looking healthy.

CASE II.-Mrs. - aged 30. Was always healthy previous to her confinement, which was natural, in the spring of 1864 . Soon thereafter, she noticed an onlargement of the hypogastrium, most marked on the right side. This had very rapidly increasad within the three months previous to my visit, at which time, in January, 1865, I found her with an amount of abdominal dis. tention sufficient to cause much interference with the functions of the viscera, and a careful examination revealed an ovarian cyst. She had been rapidly failing in strength and flesh for several weeks, and was now entirely confined to the bed, and so.weak as to require assistance in changing her position. Her pulse was very rapid and feeble, and her geueral expression such as to give very little encouragement for an operation. She had suffered from several attacks of peritonitis, no one of which was very severe.

She decided to take the forlorn chance of an operation, which I made with the assistance of Drs. Talbot and Pettee, of Wilmington, Vt., and Drs. Charles Bliss and Frank S. Abbott, then my students. The mode of procedure was similar to that adopted in Case I. The tumor, which was multilocular, was firmly adherent in many points to the abdominal walls, intestines, stomach and liver. The pedicle (right broad ligament) was transfixed, and each half tied with a suitable ligature, after which I carried an additional one around the whole, carrying the three down through the vagina, as in the first instance.

She sustained very little shock, and we left her, three hours after, cornfortable, though very feelle. She now got anodynes pro re nata, requiring only moderate doses, and was carefully supported from the first with concentrated nourishment-quinine, wine and muriated tincture of iron. As we feared, her recovery was very slow and tedious. So low was her vitality that the external wound united slowly, the lower angle at one time re-opening, through which, as well as through the vagina, much ichorous, fotid discharge issued. Such was the condition of her blood, that her mouth and throat became aphthous, and ulcerations of the mucous surfaces occurred in various places. The septum nasi was attacked and perforated, the opening now remaining admitting the little finger. The tonics and 
stimulants were increased until heroic doses were given, to which she responded well. In addition, the abdominal cavity was thoroughly washed out once or twice daily, according to indications, with artificial serum at blood heat. This was accomplished by using a long pipe (a large catheter answers nicely) attached to an elastic syringe and inserted into the opening in Douglass's cul de sac, through which the ligatures passed, which allowed the fluid ready exit after it was injected, although for a part of the time a portion of it escaped through the lower angle of the external wound.

$I$ advised Dr. 'Talbot, the attending physician, to consider symploms of peritonitis indications for this washing out of the abdominal cavity, and it invariably had the immediate effect to relieve local distress, allay fever and restlessness, and in every way improve the condition of the patient. While the discharges were of an acrid or offensive character, solutions of chlorine were added. After a tedious illness, this lady made a a good recovery, and is now in perfect health. The tumor weighed 38 pounds.

Too much praise cannot be awarded to my friend Dr. J. H. Talbot for his faithful, skilful care of this case; and to his ready appreciation of the varying indications and the prompt, energetic and skilful manner in which he met them, does this patient owe her recovery.

[To be continued.]

\section{gifospital äeprorts.}

\section{MASSACHUSETTS GENERAL HOSPITAL.}

Surgical Operations for the week ending March 28th. Reported by Messrs. Thomas Wateman, Jr. and H. H. A. BEACH.

1. Necrosis of Ilium. Dr. R. M. Hodges. -Female, aged 19. Two years ago, this patient received a bruise over her right hip from a rod. Soreness alone followed, lasting about three weeks. One year later, a small abscess pointed at this spot, and, evacuating itself, continued to discharge for two months, when it closed and remained so till two months ago, when the abscess repeated itself. There has been no pain and no lameness. On examination, there was found an irregular ulcer, about half an inch in diameter, and two inches back of the anterior superior spinous process of the right ilium; a probe introduced into this, after a little manipulation, reached diseased bone beneath. A free crucial incision was made through the ulcer down to the ilium these flaps being reflected, a sinus was found leading through the bone to the inside of the pelvis. A probe introduced through this cluaca detected diseased bone on the inside of the ilium, anterior to the point of entrance. Two disks of bone, one half an inch in diameter, were removed by the trephine-one involving the cloaca and the other three fourths of an inch anterior to it. The intervening bone was then removed by a chisel.

The inner surface of the removed bone was found roughened and a portion of its substance wanting. There was but slight hæmorrhage, and this stopped spontaneously.

2. Blepharoplasty. Dr. H. J. BIGELow.Male, aged 19. In September, 1865, this patient received a gun-shot wound. The explosion carried away the surface of the right upper lid and frontal region, leaving a considerable indentation of the bone, but without injuring the eye. On recovery, the upper lid was everted and fastened to the eyebrow, with entire loss of skin and outer two thirds of the ciliary border. The interval between the eyeball and the eyebrow was occupied by a red, granulating surface. At the first operation, the margin of the lid had been brought down from the eyebrow and a large flap, two and one half inches long and one wide, had been taken from the temple as far back as the hair. This was now cicatrized and smoothed, the pedicle having been at a previous time adjusted. Now, on the outer half of the ciliary margin the mucous membrane is still everted to the extent of one third of an inch. Operation. - The everted edge was carefully dissected and brought down, and a flap one half an inch wide and two inches and three fourths long was taken, like the last, from the temple, on a line horizontal with the eye. This was secured in the dissected interval by fine sutures.

Since the above was written, the whole flap has united, and the wound in the temple also, by the first intention.

3. Ligation of Homorrhoids. Dr. H. G. Clark. - Patient has had external hæmorrhoids for about a year. Operalion, with ether. Three double ligatures were passed through the external folds, and the skin was cut with a scalpel between the points of entrance. The ligatures were then tied in the grooves (to save time and pain in the ulceration), and the mass strangulated.

4. Tumor of Leg; Ampulation of Thigh. Dr. R. M. Hodges. - Patient, an Irish boy, aged 13. Last August, while bathing, 